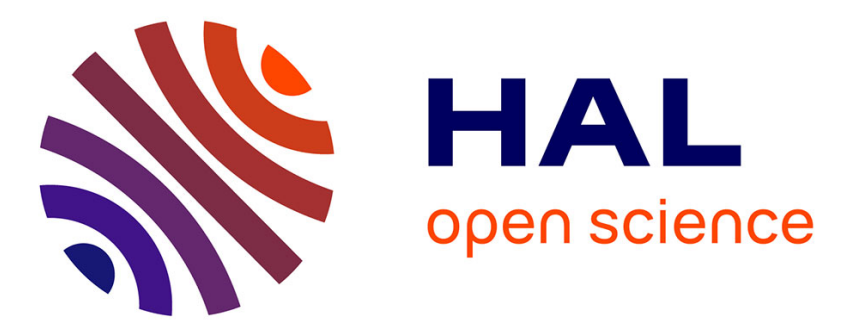

\title{
3D Visualization to Mitigate Weather Hazards in the Flight Deck: Findings from a User Study
}

Catherine Letondal, Cédric Zimmerman, Jean-Luc Vinot, Stéphane Conversy

\section{To cite this version:}

Catherine Letondal, Cédric Zimmerman, Jean-Luc Vinot, Stéphane Conversy. 3D Visualization to Mitigate Weather Hazards in the Flight Deck: Findings from a User Study. 3DUI 2015, 3DUI 2015 IEEE 10th Symposium on 3D User Interfaces, Mar 2015, Arles, France. hal-01112005

\section{HAL Id: hal-01112005 https://hal-enac.archives-ouvertes.fr/hal-01112005}

Submitted on 23 Mar 2015

HAL is a multi-disciplinary open access archive for the deposit and dissemination of scientific research documents, whether they are published or not. The documents may come from teaching and research institutions in France or abroad, or from public or private research centers.
L'archive ouverte pluridisciplinaire HAL, est destinée au dépôt et à la diffusion de documents scientifiques de niveau recherche, publiés ou non, émanant des établissements d'enseignement et de recherche français ou étrangers, des laboratoires publics ou privés. 


\title{
3D Visualization to Mitigate Weather Hazards in the Flight Deck: Findings from a User Study
}

\author{
Catherine Letondal, Cédric Zimmerman, Jean-Luc Vinot, Stéphane Conversy * \\ University of Toulouse - ENAC
}

\begin{abstract}
In this paper, we report on our exploration of $3 \mathrm{D}$ representations to support temporal navigation. We ground our discussion in a user study on the design of a tool that helps airliner pilots to manage dangerous and tall thunderstorm cells. The tool enables pilots to visualize thunderstorm cells, navigate into meteorological predictions in the near future and evaluate safe paths. The visualization is provided on the dual 2D horizontal/vertical view that is already used on the flight deck. In lieu of the standard 2D vertical view, the tool uses a 3D view that facilitates tracking of cells sliding along time slots and altitudes. The user navigates along two dimensions, heading and time slots, thanks to a rotary knob-button. The design is grounded in field studies with pilots. Prototypes have been iteratively evaluated during design walkthrough sessions with pilots. From the preliminary results of this study, we draw research questions related to the need of $3 \mathrm{D}$ in the cockpit navigation displays.
\end{abstract}

Keywords: 2D/3D, ethnography, contextual design, temporal data, aeronautics, meteorological data, in-vehicle 3D user interfaces, cockpit display.

Index Terms: H.5.2: User Interfaces. I.3.7: Three-Dimensional Graphics and Realism.

\section{INTRODUCTION}

Cumulonimbus are heavy and dense clouds of considerable vertical extent in the form of a mountain or huge tower, often associated with heavy precipitation, lightning and thunder (Fig. 1.a). Cumulonimbus can be threatening to aircraft for several reasons: vertical movements are up to 50 knots; severe icing can induce adverse aerodynamic effects, blockage of Pitot tubes and static vents and radio communication problems; electrical disturbances can effect communication and navigation systems; hail can cause significant structural damage. Airliners crossing the inter-tropical convergence zone (ICTZ), a belt of converging trade winds and rising air that encircles the earth near the equator (Fig. 2.a), are particularly exposed to rapidly developing thunderstorms (RDT).

Today, airliners are provided with meteorological data through their local weather radar. Detected area are depicted on their ND (Navigation Display) in layers of green, yellow, and red according to their increasing intensity (Fig. 1.b). While this visualization is very efficient in most cases, it has several drawbacks in areas of intense activity and bad light condition (e.g. dark moonless night): a front radar echo may hide or minimize another echo behind; determining the altitude of the echo is not straightforward; no prediction of near future weather is provided; and the radar only

* firstname.lastname@enac.fr provides a front view of 180 degrees while pilots may need to see the clouds on the sides or even at the back to evaluate possible route deviations. This is the reason why pilots are extremely interested in satellite data, that provide a "god's eye" global and almost real time view of the thunderstorm convective cells.
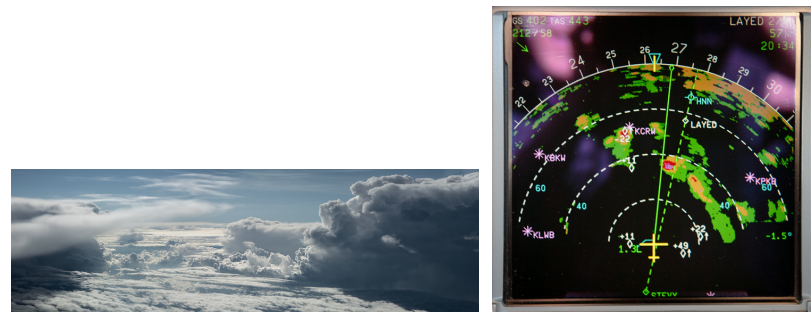

Figure 1: a) a panoramic view looking forward from the flight deck: dark clouds are towering thunderstorms. Thunderstorms are of course more dangerous in bad light conditions, during dark moonless nights; b) the navigation display of an airliner that shows the radar detection of the clouds on the left (red area indicates a definitely to avoid cumulonimbus).
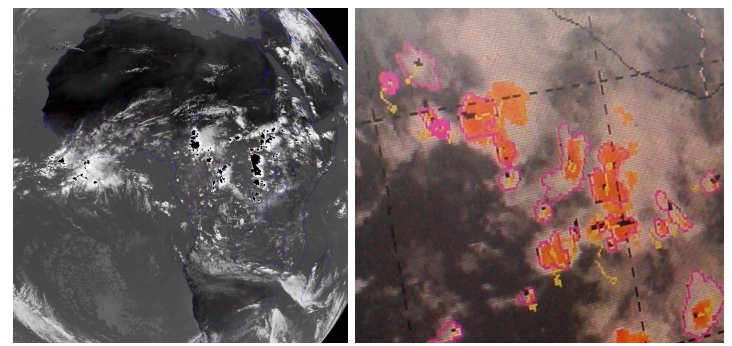

Figure 2: a) thunderstorms in the ICT zone above Africa: black colored zones are cumulonimbus up to $15 \mathrm{~km}$ high ; b) calculated RDT convective cells are superimposed in pink.

Meteorological data are today available for providing pilots with location of dangerous thunderstorm cells in the form of $3 \mathrm{D}$ data. They are acquired every 15 minutes and computed from METEOSAT satellite images (Fig 2.a). A number of algorithms such as RDT (Rapidly Developing Thunderstorms) [2] enable 1) the identification and tracking of intense convective system clouds, 2) the detection of rapidly developing convective cells, together with various information such as their motion, cooling and expansion rate, cloud top height (3D) and associated time series (Fig. 2.b), and 3) the prediction of near future cell systems. These algorithms are based on infrared data from which spatial characteristics (top and bottom levels, peripheral gradient, surfaces), and temporal characteristics (cooling rate, parameters trend) can be determined. The results can be sent in real time to the aircraft through ground-air communication channels.

Benefits of 3D displays in the cockpit have been widely studied in the past. Several studies such as Alexander et al. [1] balance $2 \mathrm{D}+2 \mathrm{D}$ coplanar and vertical displays with $3 \mathrm{D}$ perspective views, but results do not show a strong benefit for the one or the other. Thus, instead of using a 3D view, pilots rely on this " $2 \mathrm{Dh}+2 \mathrm{Dv}$ " view, composed from an horizontal slice (or cut) and a vertical 
one, through the Vertical Situation Display (Fig 5.a), that has been shown to be less ambiguous than 3D perspective view, even if more demanding in terms of visual scanning.

We conducted a user study with pilots to explore the use of 3D visualization of thunderstorms. Starting from a $2 \mathrm{Dh}+2 \mathrm{Dv}$ view to visualize satellite-based data around the aircraft, the study has highlighted pilots' interest in such observed data. However, pilots have shown even more the interest in the estimation of alternative routes using predicted data that provide convective cell detections for the next 30 minutes. We thus explored and evaluated with pilots a design enabling to explore these predicted data, by relying on a rotary Powermate button (Fig 8.a). This enabled us to observe that a 3D design may provide a better way to follow predicted cloud moves in either altitude or angle. This design study, in contrast with results from previous research on cockpit displays, seems to clearly argue for the benefit of a 3D representation. Based on this insight, our preliminary results contribute by refining research questions related to what triggers the need of 3D in the cockpit navigation displays.

Following a related work and a methodology sections, we first describe the initial study, then a $2 \mathrm{Dh}+2 \mathrm{Dv}$ prototype and its evaluation. Next, we describe the second $3 \mathrm{D}$ prototype, and its evaluation with a pilot. A final section formulates research questions related to triggers to $3 \mathrm{D}$ in cockpit navigation displays.

\section{Related Work}

This work addresses 3D representation support to evaluate safe trajectories through a displayed map of convective cells in the cockpit of an aircraft. As such, it first pertains to the general domain of visualization that compares the benefit of 3D over 2D representations. More specifically, cognitive studies such as Alexander et al. [1] have been published that balance 2D and 3D map representations for the navigation display in the cockpit, but these studies do not include temporal navigation in near future weather situations. Our research departs from 3D virtual navigation in the sense that our virtual representation only supports and supplements the actual moves in real space. This research is also related to mobile and in-vehicle displays for spatial interaction [5], where 3D representations have been proposed $[8,9]$. However, spatial interaction in the cockpit is different: it is the aircraft that is moving in navigation space, not the user. So, no embodied interaction is possible to capture user intent as in Oulasvirta et al. [8], and less cognitive mapping of virtual to real view is involved, since the alignment of the display and the route is provided. Finally, compared to automotive 3D UI, such as the one addressed in Tönnis et al. [9], a different type of attention is required from the user, at least for the driver, since the driver has to constantly look at the road.

\section{Methodology}

Our 1-year study followed a contextual design methodology [3]. We first met meteorologists from Météo France specialized in convective cell detection and now- or fore-casting for aeronautics.

\subsection{User studies}

User studies were conducted as follows. We first interviewed a pilot from Air France in his office; this 1-hour interview was recorded and transcribed. Based on this interview, we designed the first 2D prototype that we showed to a team of 5 Air France pilots, including our first pilot, during a $1 \mathrm{~h}$ meeting that was also recorded. A $2 \mathrm{~h}$ full-flight simulator session followed, where we were able to observe the use of the navigation display using a rotary button, to interact with it. After this meeting, where the importance of evaluating cloud predictions was highlighted, a second prototype, based on $3 \mathrm{D}$ visualization and the use of a
Powermate rotary-button, has been developed. Finally, this prototype has been evaluated with the pilot of the initial study during a 1-hour session. Both interviews with this pilot (initial and final ones) included some brainstorming and prototyping phases.

It has to be noted that our design mainly relies on a single subject user study, namely the Air France pilot that we met three times over 1 year. Finding pilots is indeed quite difficult, especially pilots having experienced cross-equator flights: they are the only ones affected by rapidly developing huge cumulonimbus systems when they fly across the ICTZ. For other pilots, the plane radar does the job quite well.

\subsection{Prototyping technology}

Following an iterative design approach, our two prototypes were not fully functional: they were aimed at gathering and refining requirements from the pilots and at building a design space. So, for rapid prototyping purposes, both prototypes are based on 1) javascript running in a web navigator, 2) SVG layers simulating the navigation display and 3) some javascript libraries such as $\mathrm{d} 3$.js or three.js to easily manage weather data formatted in json.

\section{INITIAL STUDY}
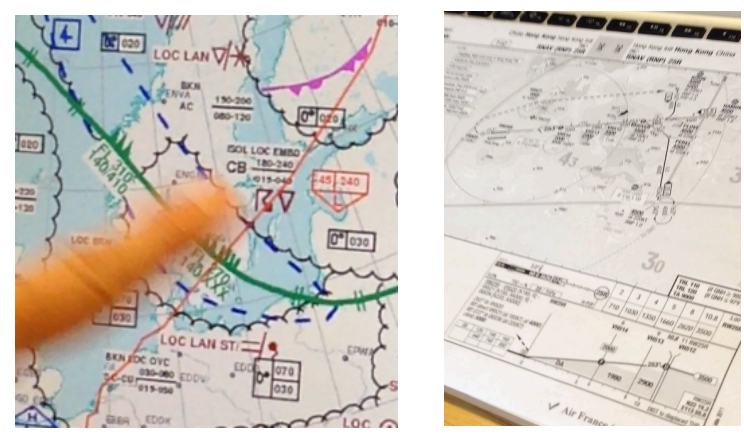

Figure 3: a) meteorological maps provided to prepare the flight; b) a dual view including a vertical profile of the route (bottom).

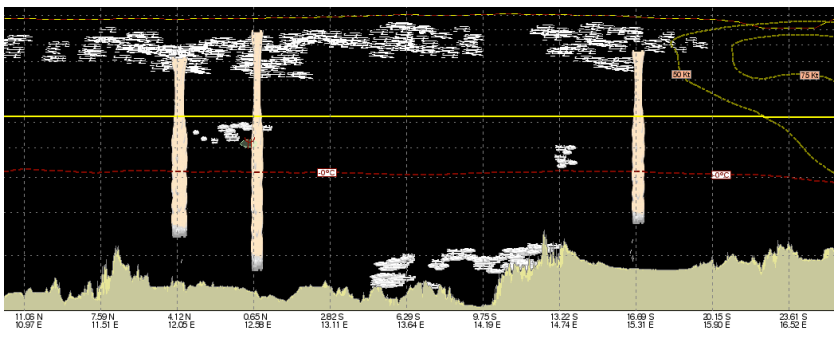

Figure 4: cumulonimbus objects (the 3 big chimneys) are highly selective in a vertical view of the route (OGIMET site [10]).

We first interviewed an Air France pilot in his office. This pilot, about 35 year old, had made numerous flights over the ICTZ (Paris-Rio, Africa, Paris-La Réunion...). This pilot also regularly takes part in studies at the Air France center in Roissy. During the interview, he showed us the documents that pilots have to study to prepare a flight and anticipate meteorological hazards (Fig 3.a). In particular, he explained that pilots are used to documents such as in Fig. 3.b that include a vertical profile of the route. He also showed an example of a website [10] displaying the terrain, the tropopause level, the wind directions and the clouds along a route in a vertical view (Fig. 4). While this view is not really made for airliner routes, pilots love it probably because cumulonimbus sizes pops-out in the visualization.

$\mathrm{He}$ also reported about particular situations, such as one flight departing from Pointe Noire in Congo, where they had to divert from north to south to avoid huge cumulonimbus. He explained 
that the meteorological center on ground sends brief information report on a fax device available in the cockpit, but that these reports only provide global information about a very wide area. Furthermore the pilots have to enter the provided area coordinates with a virtual keyboard. When asked about the actual means he used to handle this specific situation, he also reported that pilots are required to use a dedicated channel to communicate over noncontrolled area, and share dangerous spot information.

\section{FIRSt PROTOTYPE}

\subsection{Design}

The first prototype consisted of javascript code using the $\mathrm{d} 3$ library to load and draw the RDT cell polygons. As shown in Fig. $5 . b$, the SVG background represents the cockpit ND with the plane (yellow) and a route (green). We added a layer to draw actual polygons (top) and rectangles (bottom) representing convective cells as SVG paths (in red). Turning a slider (not shown) to change headings highlights cell segments intersecting the explored route (in white), and draws schematic rectangles in the bottom part (VSD) to represent convective cell heights.
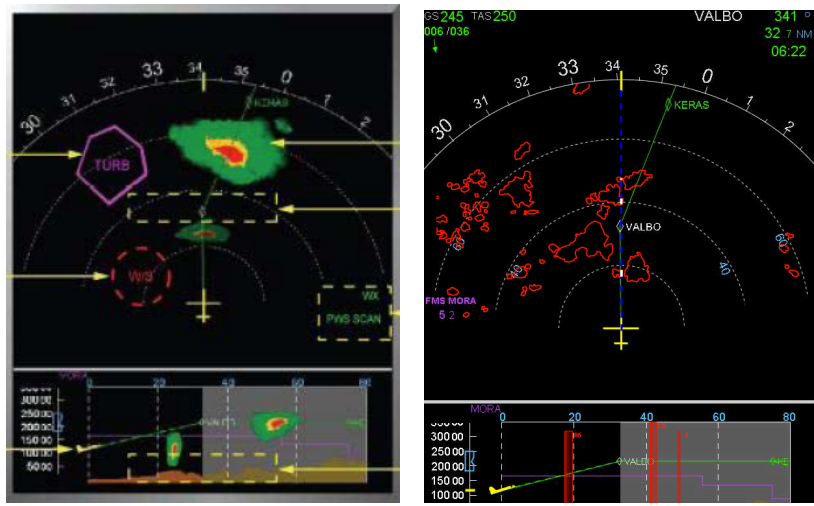

Figure 5: A) the current A380 ND, using a 2Dh+2Dv: the lower part (VSD: Vertical Situation Display) shows the vertical profile of the route; b) first prototype: we add a view of the RDT cells (in red) that intersect the "tilt" (upper, in blue), helping the pilot to select a cloud-free deviation.

\subsection{Evaluation}

We wanted to get feedback from pilots about 1) the visualization of convective cell height, drawing from the example documents shown by the pilot in the initial study and 2) about the exploration of alternative routes. This prototype was demonstrated to a team of pilots at the Air France technical center in Roissy, where they were available for a brief moment to discuss its functions and design before a simulator session.

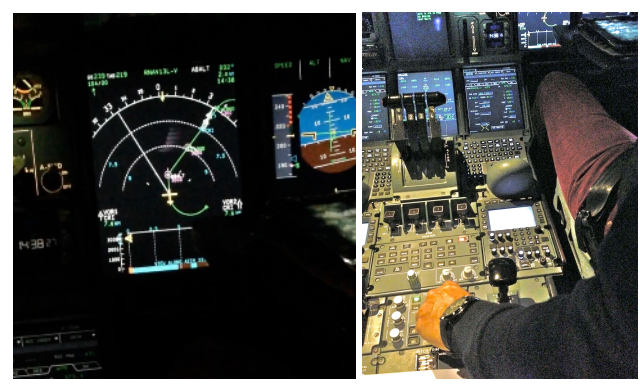

Figure 6: a) the actual A380 full-flight simulator navigation display and its "vertical cut" (white straight line); b) the pilot rotates the button to turn the vertical cut.
They all agreed that using satellite data was useful and that displaying the detected cells on the VSD was a great idea. They commented that the dashed blue line already existed in the A380 cockpit as a "vertical cut" (Fig. 6.a), and was actually associated with a physical button that sits in the middle area of the cockpit (Fig 6.b), as we were able to observe next during the full-flight simulator session. Their comment was however that, while having satellite-based detection of dangerous cells was critically important, the fact that these data were observed up to 14 minutes earlier, and thus may have moved significantly, was a real concern, as also noted in Burgess et al. [4].

\section{Second Prototype}

Our next goal was: 1) to enable the pilots to navigate within the visualization with an appropriate device, i.e. a rotary Powermate to move the "vertical cut", and 2) to provide cell predictions, since observed data were deemed almost obsolete by the pilots.

\subsection{Design}

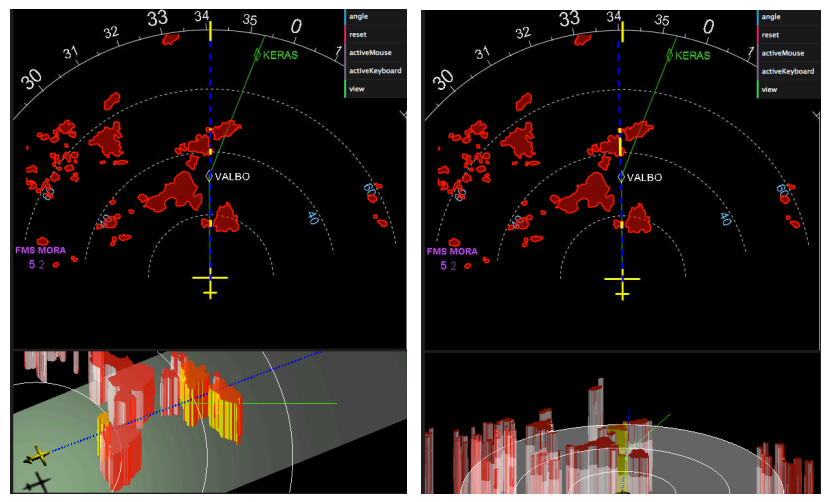

Figure 7: 2nd prototype: a) Axonometric view; b) Perspective view.

Our hypothesis for a better navigation among near future situations is that it would be easier for the pilots to follow convective cells with a $3 \mathrm{D}$ representation instead of vertical slices only. 3D shapes are drawn using three.js using a WebGL renderer. An orthographic camera was used to let the user unambiguously evaluate the dimensions of the cells, instead of a perspective camera that would change the dimensions according to the focal point. The 3D plane object has been implemented in Blender. Cells are first created as flat objects using an either red or transparent MeshPhong material, then they are extruded. In order for their outline to be easily distinguished, a top SpotLight together with a global ambient light have been added.
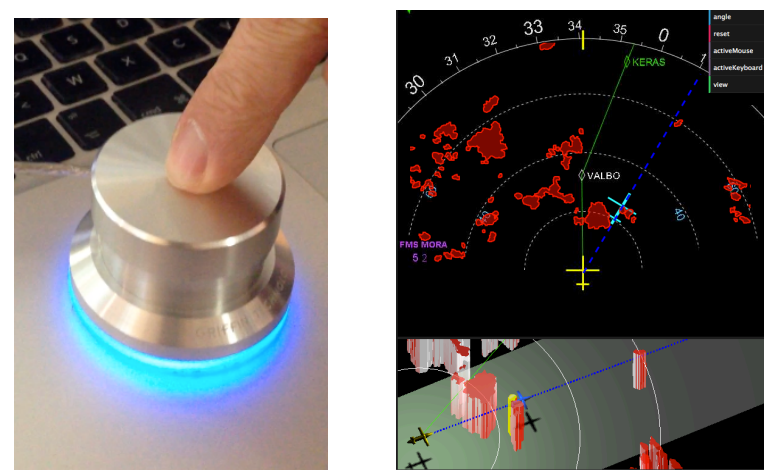

Figure 8: Second prototype: a) Powermate; b) predicting the situation in the next 15 minutes: compare with Fig. 7.a: 15 min later, the aircraft could have been able to get through given the current situation, but the cells will have moved. 
Two views with different visual properties were designed. A first, axonometric view (Fig. 7.a) preserves the apparent height of the clouds and their relative positions. The camera is located toward the plane profile and a flat area catches the shadow of the plane. A second, perspective view (Fig. 7.b) offers a subjective view to get a broader field of vision and get a better perception of the plane altitude with respect to the clouds: a horizontal circle centered on the plane cuts the clouds in half. The views were named Zaxxon and Elite according to the videogames that invented them.

We improved the control by providing a Powermate button instead of just a virtual slider (Fig. 8.a). This device has two dimensions of input: press/release and rotation. The latter interaction seemed appropriate to select a heading (an angle). In pressed mode, the pilot navigates through predictions, with each slight turn on the right projecting the plane (in blue) 15 minutes later. Powermate events are handled by a local server using node.js and forwarded to the navigator through websockets.

\subsection{Evaluation}

This second prototype was evaluated through an interview with the pilot of the initial study in a rest area at a Roissy airport terminal. The interview has been recorded and transcribed. Providing a 3D representation seemed to make sense to the pilot: he acknowledged that with 3D, you could see "on the sides", but he had no preference for either the Elite or the Zaxxon view. He commented that having animated transitions between changing shapes was not needed to better follow the clouds, particularly because an interpolation would bring additional uncertainty. He mostly commented and explored 1) the prediction mode, and 2) the Powermate device to navigate among predictions. His feedback about the availability of predictions at 15 or 30 minutes was extremely positive, together with the projection of the plane (in blue) at the anticipated corresponding location. He reflected quite a lot on how to navigate among predictions, and suggested that pressing the button several times (one for each 15 minutes prediction) (Fig. 8.a) was more consistent than rotating the button. $\mathrm{He}$ also brainstormed at length on a non-existing maintained "draw" state of the button: for more temporary visualizations, such as the "observed" cells visualization - for him not enough valuable since "in the past"; or for visualizing all thunderstorm cells whatever the flight level in the top view; or for visualizing additional information such as the quality of the predictions or other meteorological data (lightning, etc.). As observed in Letondal et al. [6], critical security and safety related functions are preferably handled by pilots through direct and physical devices. Hence the recurrent focus of the pilot on the physical aspects of the interaction with the button is for us an indication of its importance. Another recurrent comment was about the blue color (blue plane, line and button light) that is known for having a particular selectivity in the color spectrum. This design choice triggered a strong adhesion of the pilot to this "blue" mode. As he commented, "blue is the color of the future".

\section{Discussion}

Our preliminary observations seem to suggest that temporal navigation is particularly relevant to handle dangerous thunderstorms, as testified by the adhesion of the pilot. Our hypothesis is that a $3 \mathrm{D}$ representation is needed for this feature, in order to let the pilot follow the convective cells among different predictions, during a dense meteorological activity where cell levels and positions vary rapidly. Previous research, such as Alexander et al. [1], could not strongly assess the benefit of 3D for navigation displays, but did not evaluate 3D for interactively navigating along a temporal dimension. Thus, the general research questions that this study enabled us to formulate is the following: how does $2 \mathrm{Dh}+2 \mathrm{Dv}$ compare with $2 \mathrm{Dh}+3 \mathrm{D}$ ? We need to take the context into account: how do a) anticipating, exploring solutions, in particular in the presence of stress and time pressure, and b) being in nominal cruising speed, actually impact the need for 3D? In stressful conditions, an unsupported task of following 2D+2D disconnected objects may add too much cognitive load. A related question is to precisely assess the information on cells that is actually conveyed in the vertical view, and to compare it to both the horizontal and the 3D views. Indeed, while a vertical view is important to indicate the dangerousness of the clouds (depending on their height), it may not provide enough navigation support as supported through the perception of the shape and direction of the cells. Further observation and prototyping work will help to specify all these questions further. Finally, the orthographic projection requiring more matching work should be compared with a perspective projection to understand the effects of the projection on the pilots' perception of the 3D elements, and geometric enhancements as described in McGreevy et al. [7] might fix ambiguities due to the perspective projection.

\section{CONCLUSION}

Our aim in this paper is to describe a concrete problem where representing data in $3 \mathrm{D}$ has the potential of being a better solution. What we have found out is that: 1) satellite-based data are very useful for pilots to get a broader view of the situation (cell height, side and back views); 2) vertical views are potentially more informative than flat horizontal slices; 3 ) near future situations are priceless for the pilots since they enable to anticipate the actual situation at the given time and aircraft location. These findings lead us to design a tool where temporal navigation is provided, based on a 3D representation of the clouds. The results are preliminary: a thorough analysis of real situations and how the new displays can reveal (or obscure) important information is needed, as well as performance studies that show that pilots will be able to take advantage of the representations.

\section{References}

[1] Alexander, A.L. and Wickens, C.D. 2005. 3D Navigation and Integrated Hazard Display in Advanced Avionics: Performance, Aituation Awareness, and Workload. Technical Report AHFD-0510/NASA-05-2. Aviation Human Factors Division Savoy, IL.

[2] Autonès F., Moisselin, J.M. 2011. Algorithm Theoretical Basis Document for "Rapid Development Thunderstorms" (RDT/PGE11 v2.3). Technical Report SAF/NWC/CDOP/MFT/SCI/ATBD/11. Meteo France, Toulouse, France.

[3] Beyer, H. \& Holtzblatt, K. 1998. Contextual Design: Defining Customer-Centered Systems. Morgan Kaufmann.

[4] Burgess, M.A. and Thomas, R.P. 2004. The Effect of NEXRAD Image Looping and National Convective Weather Forecast Product on Pilot Decision Making in the Use of a Cockpit Weather Information Display. Technical Report NASA/CR-2004-212655. RTI International Research Triangle Park, NC, NASA, Washington.

[5] Fröhlich, P. Simon, R. Baillie, L., Roberts, J. and Murray-Smith, R. Mobile Spatial Interaction. CHI EA'07, ACM NewYork, 2841-2844.

[6] Letondal, C., Castet, J., Vinot, J.L, de la Rivière, J.B and Pauchet, S. Exploring the Physical Design Space for Situation Awareness and Performance in the Interactive Cockpit. Proc. TEI'2014 EA, ACM.

[7] McGreevy, M.W. \& Ellis, S.R. 1991. Format and Basic Geometry of a Perspective Display of Air Traffic for the Cockpit. Technical Report NASA86680. NASA Ames Research Center, CA.

[8] Oulasvirta, A., Estlander, S. and Nurminen, A. 2009. Embodied Interaction with a 3D vs 2D Mobile Map. Pers. Ubiq. Comp. 13, 4, 303-320, Springer-Verlag.

[9] Tönnis, M., Broy, V., and Klinker, G. 2006. A Survey of Challenges Related to the Design of 3D User Interfaces for Car Drivers. In Proc of 3DUI, IEEE Computer Society, Washington, DC, USA, 127-134.

[10] http://www.ogimet.com/gramet_aero.phtml.en (visited Nov. 2014). 